\title{
A Nontrivial Product in the Cohomology of the Steenrod Algebra
}

\author{
Chong Wang* \\ College of Mathematics and Statistics, Cangzhou Normal University, Hebei 061000, P. R. China. \\ * Corresponding author. Tel.: 13230737859; email: wangchong_618@163.com \\ Manuscript submitted May 14, 2017; accepted September 14, 2017. \\ doi: 10.17706/ijapm.2017.7.4.241-250
}

\begin{abstract}
Let $\mathrm{p}$ be a prime greater than seven and $\mathrm{A}$ be the mod $\mathrm{p}$ Steenrod algebra. In this paper, we prove that the product $b_{-} 0^{\wedge} 2$ delta_$_{-}\{s+4\}$ is nontrivial, where $s$ is greater than or equal to zero and less than $p-4$.
\end{abstract}

Key words: Adams spectral sequence cohomology may spectral sequence steenrod algebra.

\section{Introduction}

Homotopy groups are among the most fundamental algebraic invariants of topological spaces. To determine the homotopy groups $\pi_{*} S$ of spheres $S$ at $p$ is one of the central problems in the stable homotopy theory. Throughout this paper, $p$ will denote an arbitrary odd prime number. Let $A$ denote the mod $p$ Steenrod algebra and $S$ denote the sphere spectrum localized at an odd prime number $p$. The Adams spectral sequence $E_{2}^{s, t}=E x t_{A}^{s, t}\left(Z_{p}, Z_{p}\right) \Rightarrow \pi_{t-s} S$ has been an invaluable tool in studying the stable homotopy groups of spheres, where the $E_{2}^{s, t}$ - term is the cohomology of $A$. If a family of homotopy generators $x_{i}$ in $E_{2}^{s, *}$ converges nontrivially in the Adams spectral sequence, then we get a family of homotopy elements $f_{i}$ in $\pi_{*} S$ and we say that $f_{i}$ is represented by $x_{i} \in E_{2}^{s, *}$ and has filtration $s$ in the Adams spectral sequence. So far, not so many families of homotopy elements in $\pi_{*} S$ have been detected. For example, R. Cohen [1] constructed a certain infinite family of elements denoted by $\zeta_{k} \in \pi_{2(p-1)\left(p^{k+1}+1\right)-3} S, \quad k \geq 1$ and $p>2$. Note that the family $\zeta_{k}$ is represented by $h_{0} b_{k} \in E x t_{A}^{3,2(p-1)\left\{p^{k+1}+1\right\}}\left(Z_{p}, Z_{p}\right)$ in the Adams spectral sequence.

Throughout this paper, we fix $q=2(p-1)$. For computing the stable homotopy groups of spheres with the classical Adams spectral sequence, we must compute the $E_{2}-$ term of the Adams spectral sequence $\operatorname{Ext}_{A}^{* * *}\left(Z_{p}, Z_{p}\right)$. There are two best methods for computing $\operatorname{Ext}_{A}^{* * *}\left(Z_{p}, Z_{p}\right):$ the May spectral sequence and the lambda algebra. The known results on $\operatorname{Ext}_{A}^{*}{ }^{*}{ }^{*}\left(Z_{p}, Z_{p}\right)$ are as follows. $\operatorname{Ext}_{A}^{0, *}\left(Z_{p}, Z_{p}\right)=Z_{p}$ by its definition. From [2], we have $\operatorname{Ext}_{A}^{1, *}\left(Z_{p}, Z_{p}\right)$ has $Z_{p}$ - basis consisting of $a_{0} \in \operatorname{Ext}_{A}^{1,1}\left(Z_{p}, Z_{p}\right)$ and $h_{i} \in \operatorname{Ext}_{A}^{1, p^{i} q}\left(Z_{p}, Z_{p}\right)$ for all $i \geq 0$ and $\operatorname{Ext}_{A}^{2, *}\left(Z_{p}, Z_{p}\right)$ has $Z_{p}-$ basis consisting of 
$\alpha_{2}, a_{0}^{2}, a_{0} h_{i}(i>0), \quad g_{i}(i \geq 0), k_{i}(i \geq 0), b_{i}(i \geq 0)$, and $h_{i} h_{j}(j \geq i+2, i \geq 0)$ whose internal degrees are $2 q+1,2, p^{i} q+1, p^{i+1} q+2 p^{i} q, 2 p^{i+1} q+p^{i} q, p^{i+1} q$, and $p^{i} q+p^{j} q$ respectively. In 1980, Aikawa [3] determined $E x t_{A}^{3, *}\left(Z_{p}, Z_{p}\right)$ by $\lambda-$ algebra.

Studying higher-dimensional cohomology of the mod $p$ Steenrod algebra $A$ is an interesting subject and studied by several authors. For example, Liu and Zhao [4] prove the following theorem.

Theorem 1.1 For $p \geq 7$ and $4 \leq s<p$, the product $h_{0} b_{0} \tilde{\delta}_{s} \neq 0$ in the classical Adams spectral sequence,

where $\tilde{\delta}_{s}$ is given in [5].

In this paper, our main result can be stated as follows.

Theorem 1.2 Let $p \geq 7$ and $4 \leq s<p$. Then in the cohomology of the mod $p$ Steenrod algebra $A, E x t_{A}^{s+8, t(s)}\left(Z_{p}, Z_{p}\right)$, the product $b_{0}^{2} \tilde{\delta}_{s+4}$ is nontrivial, where $t(s)=q\left[(s+1)+(s+4) p+(s+3) p^{2}+(s+4) p^{3}\right]+s$.

The main method of proof is the (modified) May spectral sequence, so we will recall some knowledge on the May spectral sequence in Section 2. After detecting the generators of some May $E_{1}-$ terms in Section 3, we will prove the main theorem -Theorem 1.2.

\section{The May Spectral Sequence}

In this paper, we will make use of the May spectral sequence to prove our main results. For completeness, in this section we give some knowledge on it. From [6], there is a May spectral sequence $\left\{E_{r}^{s, t, *}, d_{r}\right\}$ which converges to $\operatorname{Ext}_{A}^{s, t}\left(Z_{p}, Z_{p}\right)$ with $E_{1}-$ term

$$
E_{1}^{*, * * *}=E\left(h_{m, i} \mid m>0, i \geq 0\right) \otimes P\left(b_{m, i} \mid m>0, i \geq 0\right) \otimes P\left(a_{n} \mid n \geq 0\right)
$$

where $E($ ) is the exterior algebra, $P()$ is the polynomial algebra, and

$$
h_{m, i} \in E_{1}^{12\left(p^{m}-1\right) p^{i}, 2 m-1}, b_{m, i} \in E_{1}^{2,2\left(p^{m}-1\right) p^{i+1}, p(2 m-1)}, a_{n} \in E_{1}^{1,2 p^{n}-1,2 n+1} .
$$

One has

$$
d_{r}: E_{r}^{s, t, u} \rightarrow E_{r}^{s+1 . t, u-r}
$$

and if $x \in E_{r}^{s, t, *}$ and $y \in E_{r}^{s^{\prime}, t^{\prime,}, \text { then }}$

$$
d_{r}(x \cdot y)=d_{r}(x) \cdot y+(-1)^{s} x \cdot d_{r}(y)
$$

In particular, the first May differential $d_{1}$ is given by 


$$
d_{1}\left(h_{i, j}\right)=\sum_{0<k<i} h_{i-k, k+j} h_{k, j}, d_{1}\left(a_{i}\right)=\sum_{0 \leq k<i} h_{i-k, k} a_{k}, d_{1}\left(b_{i, j}\right)=0
$$

There also exists a graded commutativity in the May spectral sequence: $x \cdot y=(-1)^{s s^{\prime}+t t^{\prime}} y \cdot x$ for $x, y=h_{m, i}, b_{m, i}$ or $a_{n}$.

For each element $x \in E_{1}^{s, t, u}$, we define $\operatorname{dim} x=s, \operatorname{deg} x=t, M(x)=u$.Then we have

$$
\left\{\begin{array}{l}
\operatorname{dim} h_{i, j}=\operatorname{dim} a_{i}=1 \\
\operatorname{dim} b_{i, j}=2 \\
\operatorname{deg} h_{i, j}=q\left(p^{i+j-1}+\ldots+p^{j}\right) \\
\operatorname{deg} b_{i, j}=q\left(p^{i+j}+\ldots+p^{j+1}\right) \\
\operatorname{deg} a_{i}=q\left(p^{i-1}+\ldots+1\right)+1 \\
\operatorname{deg} a_{0}=1 \\
M\left(h_{i, j}\right)=M\left(a_{i-1}\right)=2 i-1 \\
M\left(b_{i, j}\right)=(2 i-1) p
\end{array}\right.
$$

where $i \geq 1, j \geq 0$.

\section{Proof of the Main Theorem}

By (2.2), we know that to prove the non-triviality of the product $b_{0}^{2} \widetilde{\delta}_{s+4}$, we have to show that the representative of the product cannot be hit by any May differential. For doing it, we give the following two lemmas. The first one is a lemma on the representative of $\tilde{\delta}_{s+4}$ in the May spectral sequence.

Lemma 3.1 For $p \geq 7$ and $0 \leq s<p-4$. Then the fourth Greek letter element $\tilde{\delta}_{s+4} \in \operatorname{Ext}_{A}^{s+4, t_{1}(s)}\left(Z_{p}, Z_{p}\right)$ is represented by

$$
a_{4}^{s} h_{4,0} h_{3,1} h_{2,2} h_{1,3} \in_{1}^{s+4, t_{1}(s)+s, *}
$$

in the $E_{1}$-term of the May spectral sequence, where $\tilde{\delta}_{s+4}$ is actually $\tilde{\alpha}_{s+4}^{(4)}$ described in [7] and

$$
t_{1}(s)=q\left[(s+1)+(s+2) p+(s+3) p^{2}+(s+4) p^{3}\right]
$$

Lemma 3.2 Let $p \geq 7$ and $0 \leq s<p-4$. Then we have the May $E_{1}-$ term

$$
E_{1}^{s+7, t(s), *}=Z_{p}\left\{G_{1}, G_{2}, \ldots, G_{7}\right\}
$$

where $t(s, n)=q\left[(s+1)+(s+4) p+(s+3) p^{2}+(s+4) p^{3}\right]+s$, and 


$$
\begin{aligned}
& G_{1}=a_{4}^{s-1} a_{3} b_{3,0}^{2} h_{4,0} h_{3,1} h_{1,3}, G_{2}=a_{4}^{s} b_{3,0} b_{1,0} h_{4,0} h_{3,1} h_{1,3}, G_{3}=a_{4}^{s} b_{3,0} b_{1,2} h_{4,0} h_{3,1} h_{1,1}, G_{4}=a_{4}^{s} b_{3,0}^{2} h_{4,0} h_{1,3} h_{1,1}, \\
& G_{5}=a_{4}^{s-1} a_{2} b_{3,0} h_{4,0} h_{3,1} h_{2,2} h_{1,3} h_{1,1}, G_{6}=a_{4}^{s} b_{1,0} h_{4,0} h_{3,1} h_{2,2} h_{1,3} h_{1,1}, G_{7}=a_{4}^{s} a_{2} b_{1,2} h_{4,0} h_{3,1} h_{2,1} h_{1,3} h_{1,1} .
\end{aligned}
$$

Proof Consider $h=x_{1} x_{2} \ldots x_{m} \in E_{1}^{s+7, t(s), *}$ in the MSS, where $x_{i}$ is one of $h_{r, j}, b_{u, z}$ or $a_{k}, 0 \leq k \leq 4,0 \leq r+j \leq 4,0 \leq u+z \leq 3, r>0, j \geq 0, u>0, z \geq 0$.

$\operatorname{By}(2.5)$, we can assume that $\operatorname{deg} x_{i}=q\left(c_{i, 3} p^{3}+c_{i, 2} p^{2}+c_{i, 1} p+c_{i, 0}\right)+e_{i}$, where $c_{i, j}=0$ or $1, e_{i}=1$ if $x_{i}=a_{k_{i}}$, or $e_{i}=0$. It follows that $\operatorname{dim} h=\sum_{i=1}^{m} \operatorname{dim} x_{i}=s+7$ and

$$
\begin{gathered}
\operatorname{deg} h=\sum_{i=1}^{m} \operatorname{deg} x_{i}=q\left[\left(\sum_{i=1}^{m} c_{i, 3}\right) p^{3}+\left(\sum_{i=1}^{m} c_{i, 2}\right) p^{2}+\left(\sum_{i=1}^{m} c_{i, 1}\right) p+\left(\sum_{i=1}^{m} c_{i, 0}\right)\right]+\left(\sum_{i=1}^{m} e_{i}\right) \\
=q\left[(s+4) p^{3}+(s+3) p^{2}+(s+4) p+(s+1)\right]+s
\end{gathered}
$$

Note that $\operatorname{dim} h_{i, j}=\operatorname{dim} a_{i}=1, \operatorname{dim} b_{i, j}=2$ and $0 \leq s<p-4$. From $\operatorname{dim} h=\sum_{i=1}^{m} \operatorname{dim} x_{i}=s+7$, we can have $m \leq s+7 \leq p+2$.

Using $0 \leq s, s+1, s+3, s+3, s+4<p$ and the knowledge on the $p$ - adic expression in number theory, we have that

$$
\left\{\begin{array}{l}
\sum_{i=1}^{m} e_{i}=s ; \\
\sum_{i=1}^{m} c_{i, 0}=s+1 ; \\
\sum_{i=1}^{m} c_{i, 1}=s+4 ; \\
\sum_{i=1}^{m} c_{i, 2}=s+3 ; \\
\sum_{i=1}^{m} c_{i, 3}=s+4 .
\end{array}\right.
$$

By $c_{i, 3}=0$ or 1 , one has $m \geq s+4$ from $\sum_{i=1}^{m} c_{i, 3}=s+4$. Note that $m \leq s+7$. Thus $m$ may equal $s+4, s+5, s+6$,or $s+7$.Since $\sum_{i=1}^{m} e_{i}=s, \operatorname{deg} h_{i, j} \equiv 0(\bmod q)(i>0, j \geq 0), \operatorname{deg} a_{i} \equiv 1(\bmod q)(i \geq 0)$ and $\operatorname{deg} b_{i, j} \equiv 0(\bmod q)(i>0, j \geq 0)$, then by the graded commutativity of $E_{1}^{*, * * *}$ and degree reasons, we can assume that $h=a_{0}^{x} a_{1}^{y} a_{2}^{z} a_{3}^{k} a_{4}^{l} h^{\prime} \quad$ with $\quad h^{\prime}=x_{s+1} x_{s+2} \ldots x_{m}$, where 
$0 \leq x, y, z, k, l \leq s, x+y+z+k+l=s$.

Consequently, we have $h^{\prime}=x_{s+1} x_{s+2} \ldots x_{m} \in E_{1}^{7, t_{2}(s), *}$, where

$$
t_{2}(s)=q\left[(s+4-l) p^{3}+(s+3-l-k) p^{2}+(s+4-l-k-z) p+(s+1-l-k-z-y)\right] .
$$

From (3.1) we have

$$
\begin{aligned}
& \sum_{i=s+1}^{m} e_{i}=0 \\
& \sum_{i=s+1}^{m} c_{i, 0}=s+1-l-k-z-y \\
& \sum_{i=s+1}^{m} c_{i, 1}=s+4-l-k-z \\
& \sum_{i=s+1}^{m} c_{i, 2}=s+3-l-k \\
& \sum_{i=s+1}^{m} c_{i, 3}=s+4-l
\end{aligned}
$$

Case 1. $m=s+4$. Form $\sum_{i=s+1}^{s+4} c_{i, 3}=s+4-l$ in (3.2), we have that $l=s+4-\sum_{i=s+1}^{s+4} c_{i, 3} \geq s$. Note that $0 \leq l \leq s$. Thus $l=s$ and $x=y=z=k=0$. By (3.2), $h^{\prime}=x_{s+1} x_{s+2} x_{s+3} x_{s+4} \in E_{1}^{7, q\left(4 p^{3}+3 p^{2}+4 p+1\right),{ }^{*}}$. In this case, $h^{\prime}$ is impossible to exist. Then $h$ doesn't exist either.

Case 2. $m=s+5$. Form $\sum_{i=s+1}^{s+5} c_{i, 3}=s+4-l$ in (3.2), we have that $l=s+4-\sum_{i=s+1}^{s+5} c_{i, 3} \geq s-1$. Thus $l=s$ or $l=s$. There are five possibilities satisfying $0 \leq x, y, z, k, l \leq s$ and $x+y+z+k+l=s$.

We list all the possibilities in the following Table 1.

Table 1. Five Possibilities Satisfying $0 \leq x, y, z, k, l \leq s$ and $x+y+z+k+l=s$

\begin{tabular}{|c|c|c|c|c|c|}
\hline$s-1$ & 0 & 0 & 1 & 0 & $\begin{array}{l}E_{1}^{7, q\left(5 p^{3}+4 p^{2}+4 p+1\right), *} \\
=Z_{p}\left\{g_{1}\right\}\end{array}$ \\
\hline
\end{tabular}

$\begin{array}{llllllll}\text { The possibility } & l & x & y & z & k & E_{1}^{7, t_{2}(s), *} & h^{\prime}\end{array}$

The 1st

$s-1 \quad 1 \quad 0 \quad 0 \quad 0 \quad E_{1}^{7, q\left(5 p^{3}+4 p^{2}+5 p+2\right), *}=0 \quad$ Nonexistence

The 2nd $\quad s-1 \quad 0 \quad 1 \quad \begin{array}{llllll}7, q\left(5 p^{3}+4 p^{2}+5 p+1\right),{ }^{*} & =0 & 0 & \text { Nonexistence }\end{array}$

The 3rd 


\begin{tabular}{|c|c|c|c|c|c|c|c|}
\hline The 4th & $s-1$ & 0 & 0 & 0 & 1 & $E_{1}^{7, q\left(5 p^{3}+3 p^{2}+4 p+1\right), *}=0$ & Nonexistence \\
\hline The 5th & $s$ & 0 & 0 & 0 & 0 & $\begin{array}{l}E_{1}^{7, q\left(4 p^{3}+3 p^{2}+4 p+1\right), *} \\
=Z_{p}\left\{g_{2}, g_{3}, g_{4}\right\}\end{array}$ & $\begin{array}{l}h^{\prime}=g_{2}, g_{3}, g_{4} \\
\text { uptosign }\end{array}$ \\
\hline
\end{tabular}

In the table, $b_{3,0}^{2} h_{4,0} h_{3,1} h_{1,3}, b_{3,0} b_{1,0} h_{4,0} h_{3,1} h_{1,3}, b_{3,0} b_{1,2} h_{4,0} h_{3,1} h_{1,1}, b_{3,0}^{2} h_{4,0} h_{1,3} h_{1,1}$ denoted by $g_{1}, g_{2}, g_{3}, g_{4}$ respectively. Consequently, in this case up to sign $h=a_{4}^{s-1} a_{2} g_{1}, a_{4}^{s} g_{2}, a_{4}^{s} g_{3}, a_{4}^{s} g_{4}$ denoted by $G_{1}, G_{2}, G_{3}$ $G_{4}$ respectively.

Case 3. $m=s+6$. Form $\sum_{i=s+1}^{s+6} c_{i, 3}=s+4-l$ in (3.2), one has $l=s+4-\sum_{i=s+1}^{s+6} c_{i, 3} \geq s-2$. Thus $l=s-2$, $s-1$ or $s$. We list all the possibilities in the following table 2 .

Table 2. Form $\sum_{i=s+1}^{s+6} c_{i, 3}=s+4-l$ in (3.2), One Has $l=s+4-\sum_{i=s+1}^{s+6} c_{i, 3} \geq s-2$. Thus $l=s-2, s-1$ or $s$.

We List All the Possibilities

\begin{tabular}{|c|c|c|c|c|c|c|c|}
\hline The possibility & $l$ & $x$ & $y$ & $z$ & $k$ & $E_{1}^{7, t_{2}(s), *}$ & $h^{\prime}$ \\
\hline The 1st & $s-2$ & 2 & 0 & 0 & 0 & $E_{1}^{7, q\left(6 p^{3}+5 p^{2}+6 p+3\right), *}=0$ & Nonexistence \\
\hline The 2nd & $s-2$ & 0 & 2 & 0 & 0 & $E_{1}^{7, q\left(6 p^{3}+5 p^{2}+6 p+1\right), *}=0$ & Nonexistence \\
\hline The 3rd & $s-2$ & 0 & 0 & 2 & 0 & $E_{1}^{7, q\left(6 p^{3}+5 p^{2}+4 p+1\right), *}=0$ & Nonexistence \\
\hline The 4th & $s-2$ & 0 & 0 & 0 & 2 & $E_{1}^{7, q\left(6 p^{3}+3 p^{2}+4 p+1\right), *}=0$ & Nonexistence \\
\hline The 5th & $s-2$ & 1 & 1 & 0 & 0 & $E_{1}^{7, q\left(6 p^{3}+5 p^{2}+6 p+2\right), *}=0$ & Nonexistence \\
\hline The 6th & $s-2$ & 1 & 0 & 1 & 0 & $E_{1}^{7, q\left(6 p^{3}+5 p^{2}+5 p+2\right), *}=0$ & Nonexistence \\
\hline The 7th & $s-2$ & 1 & 0 & 0 & 1 & $E_{1}^{7, q\left(6 p^{3}+4 p^{2}+5 p+2\right), *}=0$ & Nonexistence \\
\hline The 8th & $s-2$ & 0 & 1 & 1 & 0 & $E_{1}^{7, q\left(6 p^{3}+5 p^{2}+5 p+1\right) * *}=0$ & Nonexistence \\
\hline
\end{tabular}




\begin{tabular}{|c|c|c|c|c|c|c|c|}
\hline The 9th & $s-2$ & 0 & 1 & 0 & 1 & $E_{1}^{7, q\left(6 p^{3}+4 p^{2}+5 p+1\right), *}=0$ & Nonexistence \\
\hline The 10th & $s-2$ & 0 & 0 & 1 & 1 & $E_{1}^{7, q\left(6 p^{3}+4 p^{2}+4 p+1\right), *}=0$ & Nonexistence \\
\hline The 11th & $s-1$ & 1 & 0 & 0 & 0 & $E_{1}^{7, q\left(5 p^{3}+4 p^{2}+5 p+2\right), *}=0$ & Nonexistence \\
\hline The 12th & $s-1$ & 0 & 1 & 0 & 0 & $E_{1}^{7, q\left(5 p^{3}+4 p^{2}+5 p+1\right), *}=0$ & Nonexistence \\
\hline The 13th & $s-1$ & 0 & 0 & 1 & 0 & $\begin{array}{l}E_{1}^{7, q\left(5 p^{3}+4 p^{2}+4 p+1\right), *} \\
=Z_{p}\left\{g_{5}\right\}\end{array}$ & $\begin{array}{l}h^{\prime}=g_{5} \\
\text { uptosign }\end{array}$ \\
\hline The 14th & $s-1$ & 0 & 0 & 0 & 1 & $E_{1}^{7, q\left(5 p^{3}+3 p^{2}+4 p+1\right),^{*}}=0$ & Nonexistence \\
\hline The 15th & $s$ & 0 & 0 & 0 & 0 & $\begin{array}{l}E_{1}^{7, q\left(4 p^{3}+3 p^{2}+4 p+1\right), *} \\
=Z_{p}\left\{g_{6}, g_{7}\right\}\end{array}$ & $\begin{array}{l}h^{\prime}=g_{6}, g_{7} \\
\text { uptosign }\end{array}$ \\
\hline
\end{tabular}

In the table, $b_{3,0} h_{4,0} h_{3,1} h_{2,2} h_{1,3} h_{1,1}, b_{1,0} h_{4,0} h_{3,1} h_{2,2} h_{1,3} h_{1,1}, b_{1,2} h_{4,0} h_{3,1} h_{2,1} h_{1,3} h_{1,1}$ denoted by $g_{5}, g_{6}, g_{7}$ respectively.

Consequently, in this case up to sign $h=a_{4}^{s-1} a_{2} g_{5}, a_{4}^{s} g_{6}, a_{4}^{s} g_{7}$ denoted by $G_{5}, G_{6}, G_{7}$ respectively. Case 4. $m=s+7$. Form $\sum_{i=s+1}^{s+7} c_{i, 3}=s+4-l$ in (3.2), one has $l=s+4-\sum_{i=s+1}^{s+7} c_{i, 3} \geq s-3$. Thus $l=s-3, s-2, s-1 \quad$ or $\quad s$ and $\quad h^{\prime}=y_{1} \ldots y_{7} \in E_{1}^{7, t_{2}(s),^{*}}$ where $y_{i}(1 \leq i \leq 7) \quad$ is in the form of $h_{r, j}, 0 \leq r+j \leq 4, r>0, j \geq 0$. When $l \leq s-1$, the coefficient of $p^{3}$ in $t_{2}(s)$ is $\geq 5$. In these case $h^{\prime}$ is impossible to exist. Then ${ }^{h}$ doesn't exist either.In the last possibility, $t_{2}(s)=4 p^{3}+3 p^{2}+4 p+1$ so $h_{4,0}, h_{3,1}$, $h_{2,2}$ and $h_{1,3}$ belong to $h^{\prime}$. Obviously, $h^{\prime}$ is impossible to exist in this case by the reason of dimension. Then $h$ doesn't exist either.

Combining Cases 1-4, we obtain that $E_{1}^{s+7, t(s), *}=Z_{p}\left\{G_{1}, \ldots, G_{7}\right\}$. This completes the proof of Lemma 3.2.

Lemma 3.3 (1) $b_{0}^{2} \widetilde{\delta}_{s+4} \in E x t_{A}^{s+8, t(s)}\left(Z_{p}, Z_{p}\right)$ is represented by $b_{1,0}^{2} a_{4}^{s} h_{4,0} h_{3,1} h_{2,2} h_{1,3} \in E_{1}^{s+8, t(s),{ }^{*}}$ in the MSS, where $t(s)=q\left[(s+4) p^{3}+(s+3) p^{2}+(s+4) p+(s+1)\right]+s$.

(2) For the seven generators of $E_{1}^{s+7, t(s), *}$, we have that

$$
\begin{gathered}
M\left(G_{1}\right)=M\left(G_{4}\right)=10 p+9 s+9, M\left(G_{2}\right)=M\left(G_{3}\right)=6 p+9 s+13, \\
M\left(G_{5}\right)=5 p+9 s+13, M\left(G_{6}\right)=M\left(G_{7}\right)=p+9 s+17 .
\end{gathered}
$$


Moreover, we have that $M\left(b_{1,0}^{2} a_{4}^{s} h_{4,0} h_{3,1} h_{2,2} h_{1,3}\right)=2 p+9 s+16$.

Now we give the proof of Theorem 1.2.

Proof of Theorem 1.2 From Lemma $3.3(1), b_{0}^{2} \tilde{\delta}_{s+4} \in E x t_{A}^{s+8, t(s)}\left(Z_{p}, Z_{p}\right)$ is represented by

$$
b_{1,0}^{2} a_{4}^{s} h_{4,0} h_{3,1} h_{2,2} h_{1,3} \in E_{1}^{s+8, t(s), 2 p+9 s+16}
$$

in the MSS. Now we will show that nothing hits the permanent cycle $b_{1,0}^{2} a_{4}^{s} h_{4,0} h_{3,1} h_{2,2} h_{1,3}$ under the May differential $d_{r}$ for $r \geq 1$. From Lemma 3.2, we have $E_{1}^{s+7, t(s),{ }^{*}}=Z_{p}\left\{G_{1}, G_{2}, \ldots, G_{7}\right\}$.

For the generators $G_{1}$ and $G_{4}$ whose May filtration are $M\left(G_{1}\right)=M\left(G_{4}\right)=10 p+9 s+9$ (see Lemma3.3), by the reason of May filtration, from (2.2) we see that

$$
b_{1,0}^{2} a_{4}^{s} h_{4,0} h_{3,1} h_{2,2} h_{1,3} \in E_{1}^{s+8, t(s), 2 p+9 s+16}
$$

which represents $b_{0}^{2} \tilde{\delta}_{s+4} \in E x t_{A}^{s+8, t(s)}\left(Z_{p}, Z_{p}\right)$ in the MSS is not in $d_{1}\left(E_{1}^{s+7, t(s), 10 p+9 s+9}\right)$.

Now we will show $E_{r}^{s+7, t(s), 10 p+9 s+9}=0$ for $r \geq 2$. By an easy calculation, from (2.3) and (2.4) one can have the first May differentials of $G_{1}$ and $G_{4}$ as follows:

$$
\begin{gathered}
d_{1}\left(G_{1}\right)=(-1)^{s+6} a_{4}^{s-1} a_{2} b_{3,0}^{2} h_{3,1} h_{2,2} h_{2,0} h_{1,3}+\ldots \neq 0 \\
d_{1}\left(G_{4}\right)=(-1)^{s+6} a_{4}^{s} b_{3,0}^{2} h_{2,2} h_{2,0} h_{1,3} h_{1,1}+\ldots \neq 0 .
\end{gathered}
$$

It is easy to see that the first May differentials of $G_{1}$ and $G_{4}$ are linearly independent. Consequently, the cocycle of $E_{1}^{s+7, t(s), 10 p+9 s+9}$ must be zero. This means that $E_{r}^{s+7, t(s), 10 p+9 s+9}=0$ for $r \geq 2$, from which we have that

$$
b_{1,0}^{2} a_{4}^{s} h_{4,0} h_{3,1} h_{2,2} h_{1,3} \notin d_{r}\left(E_{r}^{s+7, t(s), 10 p+9 s+9}\right) \text { for } r \geq 2 .
$$

In all, $b_{1,0}^{2} a_{4}^{s} h_{4,0} h_{3,1} h_{2,2} h_{1,3} \notin d_{r}\left(E_{r}^{s+7, t(s), 10 p+9 s+9}\right)$ for $r \geq 1$.

For the generators $G_{2}$ and $G_{3}$ whose May filtration are $M\left(G_{2}\right)=M\left(G_{3}\right)=6 p+9 s+13$ (see Lemma3.3), by the reason of May filtration, from (2.2) we see that

$$
b_{1,0}^{2} a_{4}^{s} h_{4,0} h_{3,1} h_{2,2} h_{1,3} \in E_{1}^{s+8, t(s), 2 p+9 s+16}
$$

which represents $b_{0}^{2} \tilde{\delta}_{s+4} \in E x t_{A}^{s+8, t(s)}\left(Z_{p}, Z_{p}\right)$ in the MSS is not in $d_{1}\left(E_{1}^{s+7, t(s), 6 p+9 s+13}\right)$. Now we will show 


$$
E_{r}^{s+7, t(s), 6 p+9 s+13}=0 \text { for } r \geq 2
$$

By an easy calculation, from (2.3) and (2.4) one can have the first May differentials of $G_{2}$ and $G_{3}$ as follows.

$$
\begin{aligned}
& d_{1}\left(G_{2}\right)=(-1)^{s+6} a_{4}^{s} b_{3,0} b_{1,0} h_{3,1} h_{2,2} h_{2,0} h_{1,3}+\ldots \neq 0, \\
& d_{1}\left(G_{3}\right)=(-1)^{s+6} a_{4}^{s} b_{3,0} b_{1,2} h_{3,1} h_{2,2} h_{2,0} h_{1,1}+\ldots \neq 0 .
\end{aligned}
$$

It is easy to see that the first May differentials of $G_{2}$ and $G_{3}$ are linearly independent. Consequently, the cocycle of $E_{1}^{s+7, t(s), 6 p+9 s+13}$ must be zero. This means that $E_{r}^{s+7, t(s), 6 p+9 s+13}=0$ for $r \geq 2$, from which we have that

$$
b_{1,0}^{2} a_{4}^{s} h_{4,0} h_{3,1} h_{2,2} h_{1,3} \notin d_{r}\left(E_{r}^{s+7, t(s), 6 p+9 s+13}\right) \text { for } r \geq 2 .
$$

In all, $b_{1,0}^{2} a_{4}^{s} h_{4,0} h_{3,1} h_{2,2} h_{1,3} \notin d_{r}\left(E_{r}^{s+7, t(s), 6 p+9 s+13}\right)$ for $r \geq 1$.

For the generator $G_{5}$ with May filtration $M\left(G_{5}\right)=5 p+9 s+13$, by an easy calculation, from (2.3) and (2.4) we have the first May differentials of $G_{5}$ as follows:

$$
d_{1}\left(G_{5}\right)=(-1)^{s+6} a_{4}^{s-1} a_{0} b_{3,0} h_{4,0} h_{3,1} h_{2,2} h_{2,0} h_{1,3} h_{1,1}+\ldots \neq 0 .
$$

Thus $E_{r}^{s+7, t(s), 5 p+9 s+13}=0$ for $r \geq 2$. At the same time, we also have that up to nonzero scalar

$$
d_{1}\left(G_{5}\right) \neq b_{1,0}^{2} a_{4}^{s} h_{4,0} h_{3,1} h_{2,2} h_{1,3} .
$$

In summary, $b_{1,0}^{2} a_{4}^{s} h_{4,0} h_{3,1} h_{2,2} h_{1,3} \notin d_{r}\left(E_{r}^{s+7, t(s), 5 p+9 s+13}\right)$ for $r \geq 1$.

For the generators $G_{6}$ and $G_{7}$ whose Mayfiltration are $M\left(G_{6}\right)=M\left(G_{7}\right)=p+9 s+17$ (see Lemma3.3), by the reason of May filtration, from (2.2) we see that $b_{1,0}^{2} a_{4}^{s} h_{4,0} h_{3,1} h_{2,2} h_{1,3} \in E_{1}^{s+8, t(s), 2 p+9 s+16}$ which represents $b_{0}^{2} \tilde{\delta}_{s+4} \in E x t_{A}^{s+8, t(s)}\left(Z_{p}, Z_{p}\right)$ in the MSS is not in $d_{r}\left(E_{1}^{s+7, t(s), 6 p+9 s+13}\right)$ for $r \geq 1$.

From the above discussion, we see that the permanent cycle $b_{1,0}^{2} a_{4}^{s} h_{4,0} h_{3,1} h_{2,2} h_{1,3}$ cannot be hit by any May differential in the MSS. Thus, $b_{1,0}^{2} a_{4}^{s} h_{4,0} h_{3,1} h_{2,2} h_{1,3} \in E_{1}^{s+8, t(s), 2 p+9 s+16}$ converges to $b_{0}^{2} \tilde{\delta}_{s+4} \in E x t_{A}^{s+8, t(s)}\left(Z_{p}, Z_{p}\right)$ in the MSS nontrivially. Consequently, $b_{0}^{2} \tilde{\delta}_{s+4} \neq 0$.

This finishes the proof of Theorem1.2.

\section{Acknowledgment}

The research was supported by the Youth Foundation of Hebei Educational Committee(QN2017505).We 
thank the referees for their time and comments.

\section{References}

[1] Cohen, R. (1981). Odd primary infinite families in stable homotopy theory. Mem. Amer. Math. Soc., 30(242), viii+92.

[2] Liulevicius, A. (1962). The factorizations of cyclic reduced powers by secondary cohomology operations. Memo. Amer.Math. Soc., 42, 112.

[3] Aikawa, T. (1980). 3-dimensional cohomology of the $\bmod p$ Steenrod algebra. Math. Scand, 47(1), 91-115.

[4] Liu, X., \& Zhao, H. (2009). On a product in the classical Adams spectral sequence. Proc. Amer. Math. Soc., 137(7), 2489-2496.

[5] Wang, X., \& Zheng, Q. (1998). The convergence of $\tilde{\alpha}_{s+4}^{(n)} h_{0} h_{k}$. Sci. China Ser. A, 41(6), 622-628.

[6] Ravenel, D. C. (1986). Complex Cobordism and Stable Homotopy Groups of Spheres. Academic Press, Orlando.

[7] Toda, H. (1971). On spectra realizing exterior parts of Steenord algebra. Topology, 10, 55-65.

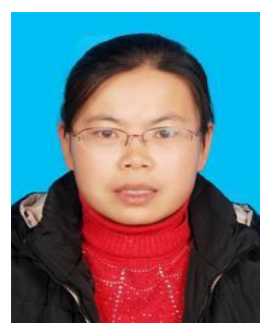

Chong Wang was born in June 1981 in Baoding, Hebei province, China. She is a lecturer. She got the master degree and graduated from Hebei Normal University in 2007, majoring in basic mathematics. Her research direction is algebraic topology and differential topology. Up to now, 14 professional papers have been published and 8 research subjects have been presided over. 